\title{
CAPACIDAD DEL TEST BASADO EN ANÁLISIS DE TRANSITORIO PARA DETECTAR FALLAS PARAMÉTRICAS
}

\section{ABILITY OF TRANSIENT ANALYSIS METHOD FOR DETECTING PARAMETRIC FAULTS}

\author{
José Peralta $^{1} \quad$ Gabriela Peretti $^{1} \quad$ Eduardo Romero $^{1,2} \quad$ Carlos Marqués $^{2}$ \\ Recibido 25 de septiembre de 2006, aceptado 5 de marzo de 2007 \\ Received: September 25, 2006 Accepted: March 5, 2007
}

\begin{abstract}
RESUMEN
En este trabajo se evalúa la capacidad de la estrategia de test denominada Método de Análisis de Transitorio para la detección de fallas paramétricas. Estas fallas son definidas como violaciones en las especificaciones de un circuito debido a desviaciones estadísticas en los componentes. Consecuentemente, el circuito es declarado como defectuoso si al menos una de las especificaciones está más allá de los límites tolerables. Un filtro de segundo orden se adopta como caso de estudio para la realización de las evaluaciones propuestas. Las especificaciones son establecidas sobre los parámetros de desempeño y los atributos de test son el tiempo de pico y la sobre-elongación de la respuesta transitoria.

Para determinar la calidad del test, son generadas 8 poblaciones de 1.000 individuos. Cada individuo es obtenido asignando un valor aleatorio para sus componentes. Las poblaciones son obtenidas mediante el incremento de la variabilidad de los componentes, desde $3 \%$ hasta $10 \%$ en pasos de $1 \%$. La evaluación concurrente de las especificaciones y de los parámetros de test permite establecer las métricas utilizadas para calificar al test. Los resultados de simulación permiten concluir que mediante la medición de los atributos de test propuestos es posible lograr un alto número de buenas decisiones de test. Adicionalmente, es posible observar que la capacidad de detección de circuitos buenos es también elevada. A pesar de estos hechos, el número de circuitos defectuosos que pasan el test podría ser inaceptable para aplicaciones que demanden una alta cobertura de fallas.
\end{abstract}

Palabras clave: Test de circuitos analógicos, método de análisis transitorio, detección de fallas paramétricas, detección de fallas de desviación.

\section{ABSTRACT}

In this work, the ability of the test strategy named Transient Analysis Method for detecting parametric faults is evaluated. These faults are defined as violations in the circuit specifications due to statistical deviations in the components. Consequently, a circuit is declared as faulty if at least one of the specifications is beyond the tolerable limits. A second order filter is adopted as a case study for performing the proposed evaluations. The specifications are established on performance parameters, and the test attributes are the peak time and the overshoot of the filter transient response.

In order to determine the test quality, 8 populations of 1.000 individuals are generated. Each individual is obtained by assigning a random value for its components. The populations are obtained by increasing the component variability from $3 \%$ up to $10 \%$ of their nominal values, in steps of $1 \%$. The concurrent evaluation of the specifications and the test parameters allow establishing the metrics used for qualifying the test. The simulation results allow concluding that by the measuring of the proposed test attributes it is possible to achieve a high number of good test decisions. Additionally, it is possible to observe that the ability for detecting good circuits is also high. Despite these facts, the number of bad circuits passing the test could be unacceptable for applications demanding high fault coverage.

Keywords: Analog circuits test, transient analysis method, parametric fault detection, deviation fault detection.

1 Grupo de Investigación y Servicios en Electrónica y Control. Facultad Regional Villa María. Universidad Tecnológica Nacional. Avda. Universidad 450,(5900) Villa María. Argentina. E-mail: gisec@frvm.utn.edu.ar

2 Grupo de Desarrollo Electrónico e Instrumental. Facultad de Matemática, Astronomía y Física, Universidad Nacional de Córdoba. Medina Allende y Haya de Torre, (5000). Córdoba, Argentina. E-mail: marques@famaf.unc.edu.ar 


\section{INTRODUCCIÓN}

Los procesos de test constituyen en la actualidad una fase fundamental en la fabricación de circuitos o sistemas electrónicos. La aplicación del test encuentra su fundamento en los inevitables errores o desviaciones en los parámetros del proceso de producción que inducen fallas en los productos, haciendo que no cumplan la función para la que han sido concebidos. Un proceso de alta calidad debe detectar lo más tempranamente posible estas partes defectuosas y evitar que las mismas lleguen al consumidor o usuario final. Las metodologías de test están orientadas a la detección y descarte de los componentes o sistemas que no cumplan con las especificaciones de diseño.

Sin embargo, este no es el único objetivo del proceso de test de sistemas electrónicos. Otra meta tan importante como la anterior es la de generar información sobre la marcha del proceso de producción para optimizar su rendimiento, pretendiéndose la disminución de la cantidad de partes rechazadas. Frecuentemente, los procedimientos en cuestión también se utilizan para la clasificación de dispositivos según el desempeño que presenten.

Actualmente se estima que la mayoría de los procedimientos de test de circuitos electrónicos incrementan los costos de producción en aproximadamente un 33\%, lo que es más que significativo. Desde este punto de vista, la presión por la disminución de los costos de producción ha sido una de las fuerzas motivadoras para el desarrollo de metodologías de test.

En particular, las secciones analógicas y de señal mixta de un sistema complejo ocupan muy poca área de silicio pero generan los mayores problemas de test. Esto se debe fundamentalmente a la naturaleza de las señales involucradas y a la baja observabilidad de los nodos internos de estos subsistemas. En términos generales puede afirmarse que para lograr un buen sistema de test de los subsistemas analógicos en un circuito de señales mixtas se requiere un esfuerzo de diseño y circuitos adicionales totalmente desproporcionados con respecto al tamaño del circuito bajo test.

Un considerable esfuerzo de investigación se ha invertido en el desarrollo de estrategias de test para los subsistemas comúnmente encontrados en las aplicaciones analógicas y de señal mixta [1,2]. Particular atención han recibido los filtros implementados en tiempo discreto o continuo. Las técnicas tradicionales para filtros se basan en verificar sus especificaciones funcionales, por ejemplo, la ubicación de los límites de la banda de paso, el desempeño en la banda de atenuación, etc. Sin embargo, este proceso demanda demasiado tiempo, impactando en el costo del proceso de test. Por esta razón aparecen técnicas alternativas, fundamentalmente orientadas a incluir el test como parte integral del diseño de estos bloques, con el objeto de disminuir el costo de este proceso [1]. Numerosas estrategias de test pueden encontrarse en la literatura, normalmente basadas en algún tipo de reconfiguración de las etapas que conforman el filtro bajo test y en el agregado de circuitos adicionales [3-7].

El Método de Análisis Transitorio (TRAM, TRansient Analysis Method) ha sido propuesto recientemente para el test de filtros de segundo orden [8-10]. La idea central de esta estrategia es excitar al sistema bajo test con una señal de entrada que provoque un transitorio subamortiguado, y asume que la presencia de fallas en el filtro provocará un cambio en el tiempo de ocurrencia del pico y en la sobre-elongación de dicho transitorio. En consecuencia, midiendo estos dos parámetros puede determinarse la presencia o no de fallas en el sistema bajo test.

La simplicidad conceptual y de implementación de TRAM, sumada a su alta eficiencia para la detección de desviaciones en los parámetros funcionales del filtro y en los valores de los componentes, la hacen sumamente atractiva tanto para implementaciones discretas como integradas.

Sin embargo, debe mencionarse que para la evaluación de la eficiencia de esta estrategia se ha utilizado el modelo de falla de desviación única en los componentes. Este modelo consiste en perturbar uno de los valores de los parámetros (componentes) del circuito mientras los demás adoptan sus valores nominales. Si bien este modelo es sencillo y se encuentra relativamente aceptado en la comunidad del test, no tiene en cuenta la variabilidad estadística natural de los valores de los componentes debida, por ejemplo, a desviaciones en los parámetros de la tecnología utilizada. Esto puede conducir a una sobreestimación de las capacidades de la estrategia.

En este trabajo se evalúa la capacidad de TRAM para la detección de violaciones en las especificaciones del filtro bajo test provocadas por variaciones estadísticas en los parámetros del circuito, denominadas fallas de tipo paramétrico. La ponderación de la estrategia se realiza para condiciones de variabilidad creciente de los valores de los componentes.

\section{MODELOS DE FALLAS PARAMÉTRICAS}

La variación estadística en los valores de los componentes de un circuito es considerada en la actualidad como una 
de las causas fundamentales de que un sistema electrónico no cumpla con las especificaciones de desempeño. Por consiguiente, los modelos de fallas catastróficas y de desviación única utilizados hasta el presente deben ser complementados con otros que permitan evaluar la eficiencia de las técnicas de test bajo hipótesis de variabilidad múltiple en los parámetros circuitales [1]. Adicionalmente, se debe mencionar que la detección de fallas debido a este tipo de variaciones es mucho más difícil que la de las catastróficas. Esto se debe a que las últimas alteran la conectividad del circuito, o bien cambian significativamente los valores de los componentes, generando cambios importantes en el comportamiento del sistema que pueden detectarse en forma relativamente simple. Por otra parte, y a diferencia de las fallas catastróficas, es difícil cuantificar el universo de fallas paramétricas posibles, dado que no puede garantizarse que un conjunto dado de variaciones provoque un error en la salida. Por ende, es necesario utilizar técnicas probabilísticas para analizar el impacto de fallas paramétricas múltiples.

Debido a lo anteriormente expuesto, existe un marcado interés en la comunidad científica por generar modelos de fallas y estrategias de simulación que permitan la evaluación de las estrategias de test considerando variaciones múltiples en los parámetros del circuito.

Los autores de [11] proponen una metodología de simulación de fallas paramétricas y establecen métricas para ponderar la eficiencia de la estrategia de test bajo estudio. Para ello sugieren dos modelos de fallas: el modelo de falla única y el modelo de falla de grupo. En el primer modelo se considera que todos los parámetros del circuito varían dentro de sus tolerancias (para estos se asume una distribución normal) y sólo uno adopta un valor fuera de tolerancia. En el segundo modelo se considera que un grupo de componentes puede sufrir desviaciones en sus tolerancias. Un modelo de falla única similar se adopta en [12], proponiéndose también un algoritmo que permite disminuir el costo computacional de la simulación de este tipo de falla. Otros autores [13] han utilizado este modelo y estudiado su relación con las especificaciones del circuito, con el objeto de remover alguna de ellas y de esa forma reducir el tiempo de test.

Savir y Guo abordan en [14] el problema de determinar las mínimas desviaciones paramétricas que pueden ser detectadas por una estrategia dada de test. Para ello asumen que se conoce la función de transferencia y su dependencia explícita con los parámetros del circuito, y determinan los valores extremos que pueden alcanzar los coeficientes de dicha función cuando todos los componentes del circuito varían simultáneamente dentro de sus tolerancias. Se asume que cualquier desviación única que provoque que un coeficiente de la función de transferencia se aparte de los límites antes determinados constituye una falla detectable. Este principio es extendido en [15] a sistemas para los cuales es imposible obtener analíticamente sus funciones de transferencia. La propuesta de los autores es obtenerlas numéricamente mediante la utilización concurrente de simulación Montecarlo y LNAPTF (Linear Network Analysis Program for Transfer Function). Finalmente en [16] proponen una metodología para la determinación de la cobertura de fallas paramétricas pequeñas.

Los autores de [17] proponen inyectar fallas en los parámetros de bajo nivel del circuito, adoptando para ello dos distribuciones gaussianas desplazadas a ambos lados de la distribución del parámetro sin fallas. La inyección se realiza en un parámetro por vez (haciendo uso de simulación Montecarlo) mientras los demás varían dentro de sus límites de tolerancia. Para cada instancia del circuito es posible determinar el cumplimiento de las especificaciones y al mismo tiempo evaluar los criterios de test para su clasificación como aceptado o rechazado. De esta forma es posible utilizar métricas de test como el rendimiento del proceso o la cobertura de fallas. Se debe mencionar que los autores definen a una falla relacionando las desviaciones en los parámetros de bajo nivel con las especificaciones. En este sentido, se considera que un circuito es defectuoso cuando las variaciones en los parámetros de bajo nivel provocan que el circuito viole por lo menos una de las especificaciones.

En [18] se adopta un modelo que asume una distribución normal para los parámetros sin fallas del circuito. Se considera que el parámetro con desviación anormal tiene una distribución uniforme que se encuentra a ambos lados de los límites de la distribución sin fallas. La detectabilidad de una falla se determina mediante el análisis de las distribuciones con y sin fallas obtenidas usando el modelo antes descrito.

Raghunathan, Chun y Abraham evalúan en [19] la estrategia denominada Test Predictivo Basado en CuasiOscilación (PQOBT, Predictive Quasi Oscillation Based Test) considerando que los parámetros del circuito tienen una distribución gaussiana. Mediante la evaluación concurrente de las especificaciones y de las predicciones de las mismas logradas mediante PQOBT, es posible determinar el grado de exactitud de la técnica para la discriminación de circuitos buenos y malos. Esta evaluación se repite para variabilidades crecientes, lo que permite determinar si la técnica mantiene su calidad en estas 
situaciones. Un enfoque similar es empleado en [20, 21] para la evaluación de esquemas OBT aplicados al test de sistemas microelectromecánicos (MEM, Micro-Electro Mechanical System). Finalmente, se debe puntualizar que la evaluación de TRAM presentada aquí se basa en principios similares a los encontrados en [19-21].

\section{CASO DE ESTUDIO: FILTRO DE SEGUNDO ORDEN}

En este trabajo se adopta como caso de estudio un filtro pasabanda de segundo orden propuesto en [22]. La topología del mismo puede verse en la figura 1.

En lo que sigue, se denominan parámetros directos a aquellos parámetros relacionados con las especificaciones funcionales. Para el filtro bajo estudio las especificaciones funcionales adoptadas son el factor de calidad del filtro, Qp, y la frecuencia de corte, Wp. Por otra parte, se denominan parámetros de bajo nivel a los componentes del circuito, resistencias y capacidades. Se debe mencionar que otras especificaciones funcionales podrían haberse elegido para el filtro bajo test, no obstante se utilizan éstas con propósitos demostrativos.

Debido a que se trata de un filtro de segundo orden, es posible obtener las expresiones (1) y (2) que relacionan los parámetros directos con los parámetros de bajo nivel:

$$
W p=\sqrt{\frac{R_{2}}{C_{2} R_{4} C_{1} R_{3} R_{5}}}
$$

$$
Q p=\sqrt{\frac{R_{2}}{C_{2} R_{4} C_{1} R_{3} R_{5}}} \cdot \frac{C_{1} R_{3} R_{5} R_{1} \cdot\left(R_{6}+R_{7}\right)}{R_{6}\left(R_{5} R_{1}+R_{5} R_{2}+R_{2} R_{1}\right)}
$$

Como fuese mencionado en la sección introductoria, TRAM establece si un circuito presenta fallas evaluando dos parámetros de la respuesta oscilatoria del circuito, el tiempo de pico ( $\mathrm{Tp}$ ) y el valor de la sobre-elongación (OS). Se denominan parámetros indirectos a estos dos atributos de test. Estos dos valores han sido señalados en la respuesta nominal a la rampa (figura 2). Las expresiones (3) y (4) relacionan los parámetros indirectos con los parámetros de bajo nivel.

$$
\begin{gathered}
T_{p}=\frac{\pi}{\sqrt{\frac{R_{2}}{C_{2} R_{4} C_{1} R_{3} R_{5}}} \cdot \sqrt{1-\zeta^{2}}} \\
O S=e^{-\pi \frac{\zeta}{\sqrt{1-\zeta^{2}}}}
\end{gathered}
$$

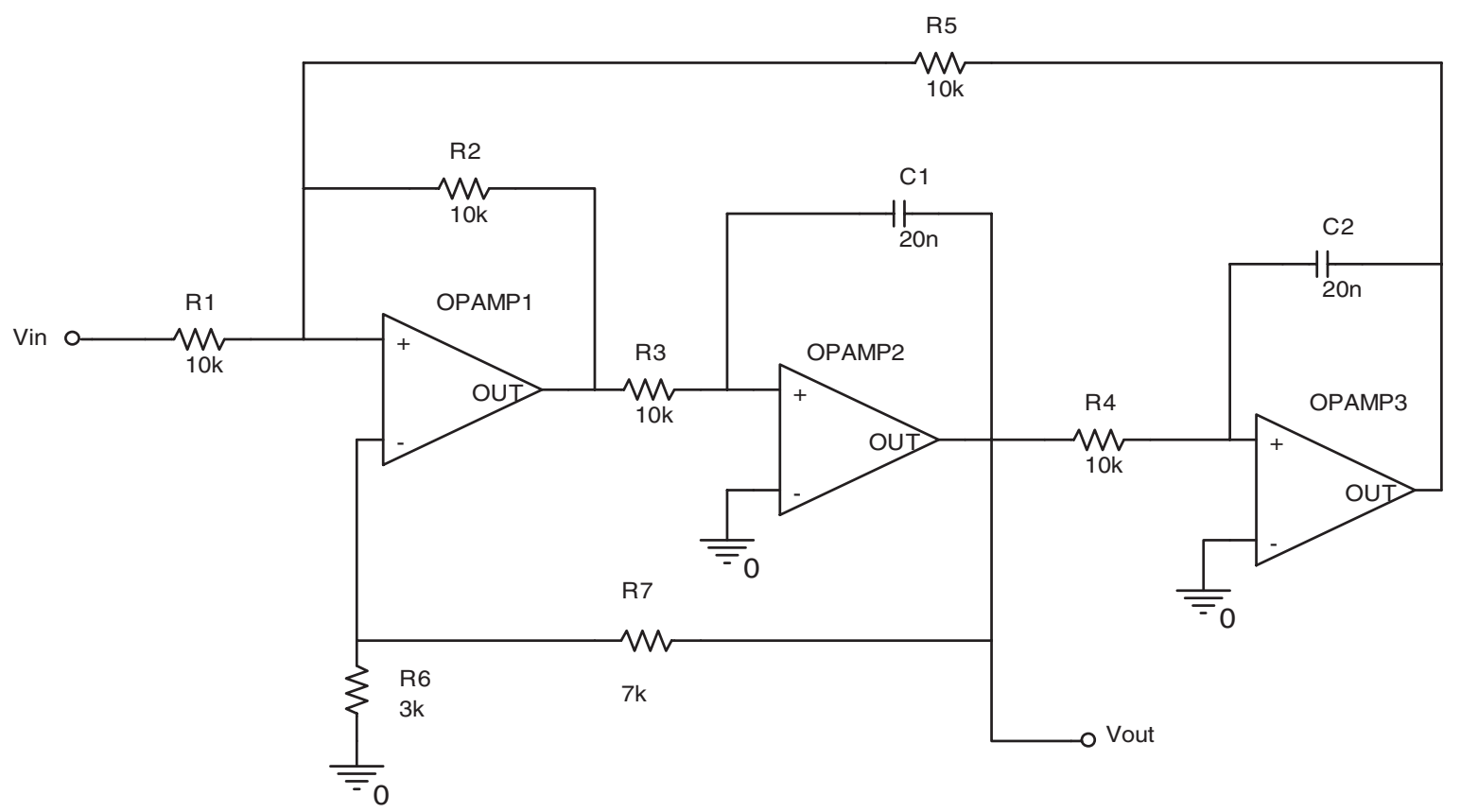

Figura 1. Filtro bajo test. 


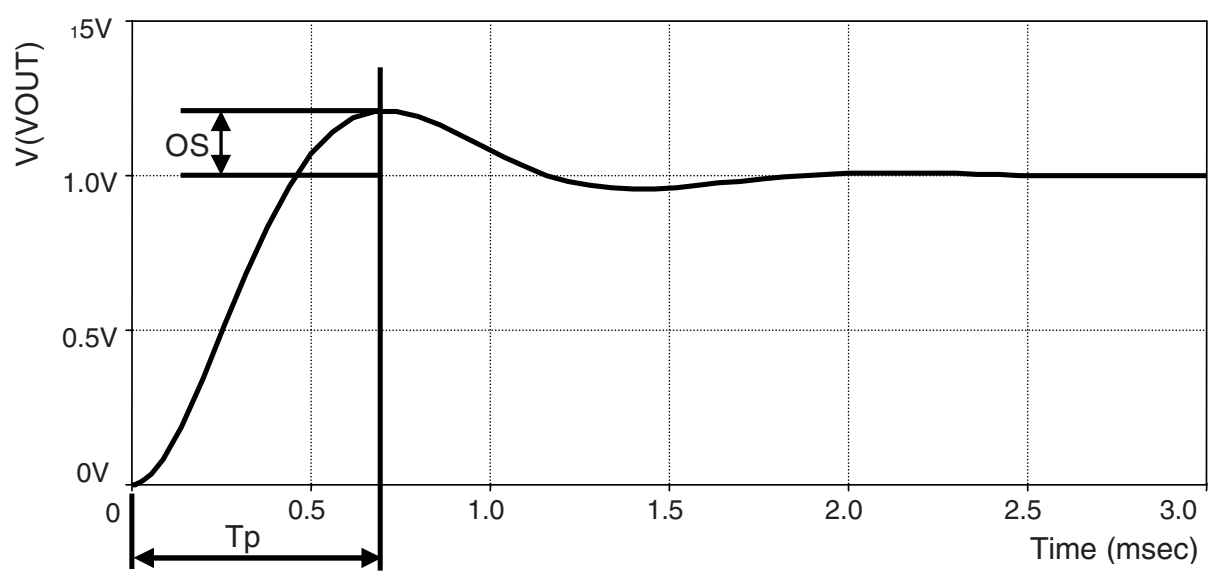

Figura 2. Respuesta nominal a la rampa.

donde, para el caso del filtro bajo estudio, $\zeta$ es dada por:

$$
\zeta=\frac{R_{6} \cdot\left(R_{5} R_{1}+R_{2} R_{5}+R_{2} R_{1}\right)}{2 \cdot C_{1} R_{3} R_{5} R_{1} \cdot\left(R_{6}+R_{7}\right)} \cdot \frac{1}{\sqrt{\frac{R_{2}}{C_{2} R_{4} C_{1} R_{3} R_{5}}}}
$$

Las expresiones anteriores son de gran importancia para la metodología de evaluación propuesta en este trabajo, ya que permiten la determinación de los parámetros necesarios para definir la calidad del test, evitando la simulación del circuito. De esta forma, es posible disminuir notoriamente el costo computacional.

\section{MODELO DE FALLA Y PROCEDIMIENTO DE EVALUACIÓN}

En este trabajo se reemplaza el modelo de falla de desviación única utilizado por otros autores, definiendo una falla como una violación a las especificaciones, independientemente de la magnitud de la variación de los parámetros de bajo nivel. Consecuentemente, se considera a un circuito como bueno si cumple simultáneamente con todas las especificaciones, mientras que si viola al menos una se considera como malo. Se pretende evaluar la capacidad de TRAM para realizar la discriminación entre ellos, bajo condiciones de aumento progresivo de la variabilidad de los componentes.

\section{Determinación de los límites de los parámetros directos}

Para el caso de estudio adoptado se definen especificaciones sobre $W p$ y $Q p$. Los valores nominales resultan de evaluar las ecuaciones (1) y (2) para los valores de R y $\mathrm{C}$ especificados en el esquemático de la Figura 1. Se admite una tolerancia de $\pm 10 \%$ en los parámetros directos, lo que permite obtener los Límites Inferior y Superior de Especificaciones (LIE y LSE) (tabla 1). Debe mencionarse que estas tolerancias dependen de la aplicación en curso y fueron fijadas arbitrariamente con fines demostrativos.

Tabla 1. Valores nominales, LIE y LSE para los parámetros directos.

\begin{tabular}{|l|r|r|}
\hline \multicolumn{1}{|c|}{ Parámetros Directos } & \multicolumn{1}{c|}{$\mathbf{W p}$} & Qp \\
\hline $\begin{array}{l}\text { Límite Inferior } \\
\text { Especificaciones (LIE) }\end{array}$ & $4.5 \mathrm{E}+03$ & 1.0000 \\
\hline Valor Nominal & $5 \mathrm{E}+03$ & 1.1111 \\
\hline $\begin{array}{l}\text { Límite Superior } \\
\text { Especificaciones (LSE) }\end{array}$ & $5.5 \mathrm{E}+03$ & 1.2222 \\
\hline
\end{tabular}

\section{Determinación de los límites de los parámetros indirectos}

En los experimentos se consideró que cada parámetro de bajo nivel presenta distribución normal, cuya media corresponde al valor nominal. Con el propósito de determinar la calidad del test bajo condiciones de aumento progresivo de la variabilidad en los componentes se generaron 8 poblaciones de 1.000 individuos cada una. Las poblaciones fueron obtenidas aumentando la variabilidad de los componentes $(\sigma)$ entre $3 \%$ y $10 \%$ del valor nominal, en pasos de $1 \%$. Un individuo de una 
población en particular se obtiene asignando un valor aleatorio para cada uno de sus componentes en el rango de variabilidad establecido para la población a la que pertenece. Para cada individuo se evalúan Wp y Qp y se lo clasifica como bueno o malo según se violen o no el LIE o el LSE. Los resultados obtenidos se muestran en la tabla 2. En esta tabla y en las que siguen se referencia cada población con el valor $\sigma$ (expresada como porcentaje del valor nominal) que se utilizó para generarla.

Tabla 2. Poblaciones obtenidas para las diferentes variabilidades consideradas.

\begin{tabular}{|c|c|c|}
\hline \multirow{2}{*}{ Población } & \multicolumn{2}{|c|}{$\begin{array}{c}\text { Clasificación según } \\
\text { especificación Wp y Qp }\end{array}$} \\
\cline { 2 - 3 } & Buenos & Malos \\
\hline $3 \%$ & 969 & 31 \\
\hline $4 \%$ & 870 & 130 \\
\hline $5 \%$ & 728 & 272 \\
\hline $6 \%$ & 603 & 397 \\
\hline $7 \%$ & 440 & 560 \\
\hline $8 \%$ & 423 & 577 \\
\hline $9 \%$ & 353 & 647 \\
\hline $10 \%$ & 281 & 719 \\
\hline
\end{tabular}

Los valores límites de los parámetros indirectos (Tp y OS) que se utilizarán en la fase operativa del test se determinan tomando sólo la población de buenos y se reportan en la tabla 3. Puede observarse en esta tabla una variabilidad despreciable de los parámetros indirectos obtenidos en las diferentes corridas. Por este motivo se decidió adoptar como límites a los correspondientes a la corrida de simulación con $\sigma=3 \%$.

Tabla 3. Límites de los parámetros indirectos para las variabilidades consideradas.

\begin{tabular}{|c|c|c|c|c|}
\hline \multirow{2}{*}{ Población } & \multicolumn{4}{|c|}{ Límites para Tp y OS } \\
\cline { 2 - 5 } & Mín Tp & Máx Tp & Mín Os & Máx Os \\
\hline $3 \%$ & $6.324 \mathrm{E}-4$ & $7.997 \mathrm{E}-4$ & $1.64 \mathrm{E}-1$ & $2.442 \mathrm{E}-1$ \\
\hline $4 \%$ & $6.335 \mathrm{E}-4$ & $7.917 \mathrm{E}-4$ & $1.632 \mathrm{E}-1$ & $2.445 \mathrm{E}-1$ \\
\hline $5 \%$ & $6.281 \mathrm{E}-4$ & $7.947 \mathrm{E}-4$ & $1.633 \mathrm{E}-1$ & $2.445 \mathrm{E}-1$ \\
\hline $6 \%$ & $6.31 \mathrm{E}-4$ & $7.97 \mathrm{E}-4$ & $1.633 \mathrm{E}-1$ & $2.443 \mathrm{E}-1$ \\
\hline $7 \%$ & $6.301 \mathrm{E}-4$ & $8.03 \mathrm{E}-4$ & $1.641 \mathrm{E}-1$ & $2.445 \mathrm{E}-1$ \\
\hline $8 \%$ & $6.316 \mathrm{E}-4$ & $8.047 \mathrm{E}-4$ & $1.632 \mathrm{E}-1$ & $2.444 \mathrm{E}-1$ \\
\hline $9 \%$ & $6.330 \mathrm{E}-4$ & $8.021 \mathrm{E}-4$ & $1.631 \mathrm{E}-1$ & $2.445 \mathrm{E}-1$ \\
\hline $10 \%$ & $6.344 \mathrm{E}-4$ & $7.98 \mathrm{E}-4$ & $1.631 \mathrm{E}-1$ & $2.445 \mathrm{E}-1$ \\
\hline
\end{tabular}

\section{Obtención de las métricas de ponderación del test}

Cada individuo es clasificado como bueno o malo, según se cumplan o no las especificaciones. Por otro lado, un circuito pasa el test cuando los parámetros indirectos se encuentran dentro de los límites establecidos previamente; falla el test en caso contrario. En consecuencia, la evaluación concurrente de los parámetros directos e indirectos para las poblaciones generadas permite establecer las siguientes métricas de ponderación:

Buenos Aceptados (BA): $\mathrm{N}^{\circ}$ de circuitos que cumplen las especificaciones y pasan el test.

Buenos Rechazados (BR): $N^{\circ}$ de circuitos que cumplen las especificaciones y fallan el test.

Malos Aceptados (MA): $\mathrm{N}^{\circ}$ de circuitos que no cumplen las especificaciones y pasan el test.

Malos Rechazados (MR): $\mathrm{N}^{\mathrm{o}}$ de circuitos que no cumplen las especificaciones y fallan el test.

Buenas Decisiones (BD): $\frac{B A+M R}{\text { total deindividuos }} \cdot 100$

La tabla 4 muestra los resultados obtenidos en función de la variabilidad asignada a los componentes. Estos resultados han sido graficados en la figura 3 de manera porcentual. Como puede observarse en esta figura, la cantidad de circuitos buenos (expresados como un porcentaje de la población total) disminuye continuamente a medida que aumenta la variabilidad de los componentes (desde el 97\% hasta el 28\%), que es un resultado esperado. La curva de buenos aceptados, obtenida como un porcentaje de los circuitos buenos, con valores cercanos al $98 \%$ promedio, muestra la elevada capacidad de los parámetros indirectos para reconocer los circuitos buenos.

Tabla 4. Métricas de ponderación del test.

\begin{tabular}{|c|c|r|r|r|c|}
\hline \multirow{2}{*}{ Población } & \multicolumn{5}{|c|}{ Métrica } \\
\cline { 2 - 6 } & BA & BR & MA & MR & BD (\%) \\
\hline $3 \%$ & 966 & 3 & 2 & 29 & 99,5 \\
\hline $4 \%$ & 865 & 5 & 17 & 113 & 97,8 \\
\hline $5 \%$ & 721 & 7 & 38 & 234 & 95,5 \\
\hline $6 \%$ & 590 & 13 & 50 & 347 & 93,7 \\
\hline $7 \%$ & 435 & 5 & 36 & 524 & 95,9 \\
\hline $8 \%$ & 411 & 12 & 45 & 532 & 94,3 \\
\hline $9 \%$ & 348 & 5 & 46 & 601 & 94,9 \\
\hline $10 \%$ & 273 & 8 & 39 & 680 & 95,3 \\
\hline
\end{tabular}




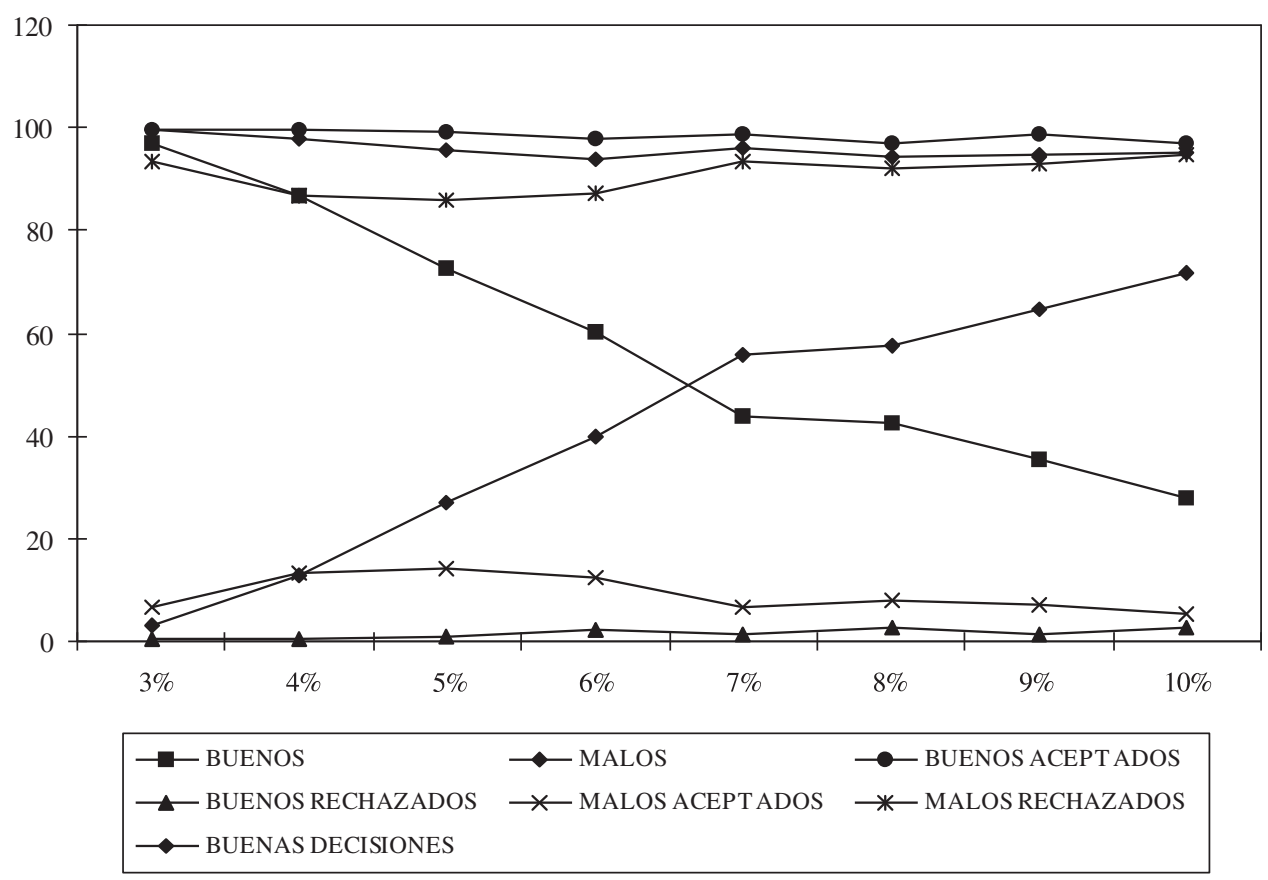

Figura 3. Resultados obtenidos.

Los malos aceptados, expresados como un porcentaje de los circuitos malos, representan los malos no reconocidos como tales a través de los parámetros indirectos. La curva respectiva muestra valores más altos a variabilidades bajas, ubicándose alrededor de $13 \%$ como promedio. Para variabilidades elevadas se alcanzan valores de aproximadamente el $7 \%$.

Finalmente, la curva de buenas decisiones (la más importante) oscila entre valores cercanos al $95 \%$ y el $99,5 \%$, lo cual indica que la eficacia de los parámetros indirectos escogidos para el test es elevada.

\section{CONCLUSIONES}

En el presente trabajo se evalúa la capacidad de TRAM para la detección de desviaciones en los parámetros funcionales de un filtro de segundo orden. A diferencia de enfoques anteriores, se define a una falla como una violación a las especificaciones de diseño, independientemente de la magnitud de las variaciones que se den en los parámetros de bajo nivel. Debe destacarse que el paradigma de falla única empleado previamente por otros autores se abandona aquí. Las evaluaciones se realizaron bajo condiciones de variabilidad creciente de los parámetros de bajo nivel del circuito.
Los resultados de simulación demuestran que TRAM logra mantener un alto porcentaje de buenas decisiones (no inferior al 95\%) en el rango de variabilidad considerado. En este sentido, los resultados son promisorios. Sin embargo, se observa un promedio de aproximadamente $13 \%$ de malos aceptados. Este puede ser un valor demasiado elevado para un gran número de aplicaciones que requieran alta eficiencia en la detección de dispositivos defectuosos (tests orientados a alta cobertura de fallas). Por otro lado, y desde el punto de vista del rendimiento del proceso, la alta capacidad de los parámetros indirectos para reconocer a los circuitos buenos se constituye en una de las cualidades más importantes de la estrategia.

\section{REFERENCIAS}

[1] B. Vinnakota (Editor). "Analog and Mixed-Signal Test”. Prentice Hall. New York, United States. 1998.

[2] A. Chatterjee, N. Nagi. "Design for testability and built-in self-test of mixed-signal circuits". Proceedings $10^{\text {th }}$ International Conference on VLSI Design, pp. 388-392. January 1997. 
[3] M. Soma. "A design-for-test methodology for active analog filters". Proceedings IEEE International Test Conference, pp. 183-192. September 1990.

[4] D. Vázquez, A. Rueda, J. Huertas. "A new strategy for testing analog filters". IEEE VLSI Test Symposium, pp. 36-41. April 1994.

[5] K. Arabi, B. Kaminska. "Oscillation-test methodology for low-cost testing of active analog filters". IEEE Transactions on Instrumentation and Measurement. Vol. 48 No 4, pp. 798-806. August 1999.

[6] E. Romero, G. Peretti, G. Huertas, D. Vázquez. "Test of switched-capacitor ladder filters using OBT". Microelectronics Journal. Vol. $36 \mathrm{~N}^{\mathrm{o}} 12$, pp. 1073-1079. December 2005.

[7] J. L. Catalano, G. Peretti, E. Romero, C. Marqués. "Exploring the ability of oscillation based test for testing continuous-time ladder filters". Proceedings of the 7th International Symposium on Quality of Electronic Design, pp. 543-550. April 2006.

[8] J. Calvano, V. Alves, M. Lubaszeswski. "Fault detection methodology for second order filters using compact test vectors transient analysis". $3^{\text {rd }}$ International Workshop on Design of Mixed-Mode Integrated Circuits and Applications, pp. 18-24. July 1999.

[9] J. Calvano, V. Alves, M. Lubaszeswski. "Fault detection methodology and BIST method for $2^{\text {nd }}$ order Butterworth, Chebyshev and Bessel Approximations". Proceedings of the $18^{\text {th }}$ IEEE VLSI Test Symposium, pp. 319-324. April 2000.

[10] J. Calvano, V. Alves, M. Lubaszeswski. “Designing for test analog signal processors for MEMS-based inertial sensors". Proceedings of the $3^{\text {rd }}$ IEEE International Workshop on System on Chip for Real Time Applications, pp. 251-256. June 2003.

[11] K. Saab, N. Ben-Hamida, B. Kaminska. "Parametric fault simulation and test vector generation". Proceedings of the Conference on Design, Automation and Test in Europe, pp. 650-656. March 2000.

[12] A. Khouas, A. Derieux. "Fault simulation for analog circuits under parameter variations". Journal of Electronic Testing: Theory and Applications. Vol. $16 \mathrm{~N}^{\circ} 3$, pp. 269-278. October 2000.
[13] S. Chang , C. Lee, J. Chen. "Structural fault based specification reduction for testing analog circuits". Journal of Electronic Testing. Vol. 18. No 6, pp. 571-581. December 2002.

[14] J. Savir, Z. Guo. "On the detectability of parametric faults in analog circuits". Proceedings of the IEEE International Conference on Computer Design: VLSI in Computers and Processors, pp. 273-276. September 2002.

[15] J. Savir, Z. Guo. "Analog circuit test using transfer function coefficient estimates". Proceedings of the IEEE International Test Conference. Vol. 1, pp. 1155-1163. September 2003.

[16] J. Savir, Z. Guo. "Test limitations of parametric faults in analog circuits". IEEE Transactions on Instrumentation and Measurement. Vol. $52 \mathrm{~N}^{\circ} 5$, pp. 1444-1454. October 2003.

[17] E. Acar, S. Ozev. "Parametric test development for RF circuits targeting physical faults locations and using specification-based fault definitions". IEEE-ACM. International Conference on Computer Aided Design, pp. 73-79. November 2005.

[18] F. Liu, S. Ozev. "Fast hierarchical process variability analysis and parametric test development for analog/ RF circuits". Proceedings of the International Conference on Computer Design, pp. 161-170. October 2005.

[19] A. Raghunathan, J. Chun y J. Abraham. "Quasioscillation based test for improved prediction of analog performance parameters". Proceedings of the International Test Conference, pp. 252-261. September 2004.

[20] A. Chaehoi, Y. Bertrand, L. Latorre, P. Nouet. "Improving the efficiency of the oscillation based test methodology for parametric faults". IEEE Latin American Test Workshop, pp. 234-237. March 2003.

[21] A. Chaehoi, L. Latorre, F. Azais, P. Nouet. "Use of a statistical approach for efficient implementation of oscillation based test strategy". Proceedings of the $9^{\text {th }}$ International Mixed Signal Test Workshop, pp. 99-103. June 2003.

[22] B. Kaminska, K. Arabi, I. Bell, P. Goteti, J. Huertas, B. Kim, A. Rueda, M. Soma. "Analog and mixed-signal benchmark circuits-First release". Proceedings of the International Test Conference, pp. 183-190. November 1997. 